\title{
Effet de la mécanisation sur la productivité des exploitations agricoles dans le Bassin arachidier au Sénégal
}

\author{
Saer Sarr, Djiby Dia, Moussa Sall, Katim Touré \& Saliou Ndiaye
}

Saer Sarr : Ingénieur agroéconomiste, Ingénieur d'études au Bureau d'analyses Macroéconomiques de l’Institut Sénégalais de Recherches Agricoles (ISRA-BAME), Sénégalais. Auteur correspondant : saer.sarr@isra.sn +221772135851

Djiby Dia : PhD Géographie, Chargé de recherches au Bureau d’analyses Macroéconomiques de l’Institut Sénégalais de Recherches Agricoles (ISRA-BAME), Sénégalais

Moussa Sall : PhD Agroéconomie, Chargé de recherches au Bureau d'analyses Macroéconomiques de l’Institut Sénégalais de Recherches Agricoles (ISRA-BAME), Sénégalaise

Katim Touré : PhD Agroéconomie, Enseignant chercheur à l’Université de Thiès, Ecole Nationale Supérieure d'Agriculture (ENSA-UT), (ISRA-BAME), Sénégalaise

Saliou Ndiaye : Pr Agronomie, Enseignant chercheur à l’Université de Thiès, Ecole Nationale Supérieure d'Agriculture (ENSA-UT), (ISRA-BAME), Sénégalaise

DOI: $10.25518 / 2295-8010.1680$

\section{Résumé :}

Cette étude a été réalisée dans le bassin arachidier du Sénégal, caractérisé par un système de rotation céréales/légumineuses en agriculture pluviale. Elle visait à analyser l'impact des équipements utilisés dans les exploitations agricoles sur les rendements des cultures. La méthodologie utilisée repose sur des enquêtes auprès de producteurs et des analyses économétriques de type log linéaire. Les résultats ont révélé un sous-équipement des exploitations agricoles dans le système pluvial. Le niveau actuel de mécanisation des exploitations a un effet positif sur la productivité de la terre mais les accroissements de rendement sont faibles et insignifiants pour l'arachide, le mil et surtout pour le maïs. L'étude a mis en évidence que, le niveau d'équipement actuel des exploitations, même doublés, ne favorise pas un accroissement significatif des rendements à l'hectare des différentes cultures dans le bassin arachidier. Cette étude renforce l'argument selon lequel, l'optimisation de l'équipement des exploitations agricoles en matériels agricoles joue un rôle déterminant dans l'expression du rendement et au final dans la productivité agricole.

\section{Abstract :}

This study was carried out in the groundnut basin of Senegal, which is characterized by a cereal/ legume rotation system in rainfed agriculture. It aimed to analyze the impact of equipment used on farms on crop yields. The methodology used is based on producer surveys and econometric analyses of the log-linear type. The results revealed an under-equipment of farms in the rainfed system. The current level of farm mechanization has a positive effect on land productivity, but yield increases are small and insignificant for groundnuts, millet and especially maize. The study showed that the current level of equipment on farms, even when doubled, does not conducive a 
Effet de la mécanisation sur la productivité des exploitations agricoles dans...

significant increase in yields per hectare of the different crops in the groundnut basin. This study reinforces the argument that the optimization of farm equipment on farms plays a determining role in the expression of yield and ultimately in agricultural productivity.

\section{Introduction}

Le poids du secteur agricole dans les économies du Nord comme du Sud ne cesse de diminuer depuis deux siècles (Berthelier et Lipchitz 2005), à la fois en termes de produit intérieur et de part de la population employée. Pourtant la production a augmenté, du fait de l'augmentation conjointe des surfaces cultivées et des rendements. Cela a été possible grâce aux innovations techniques, conçues avec l'appui de la recherche-développement et la recherche-action. Ces innovations ont concerné la mécanisation, la sélection de races animales et de semences végétales plus performantes, et le recours croissant à des intrants chimiques (engrais minéraux, produits de traitement contre les adventices, les ravageurs et les parasites). Ces innovations ont été à la base de la deuxième révolution agricole démarrée à la fin du 19ème siècle. La "Révolution verte »a désigné l'extension de ce modèle aux pays en développement, à partir des années 1960 (Mazoyer et Roudart 2008). La mécanisation agricole s'est ainsi fortement développée, en Asie et Amérique Latine et a été un facteur clé de la croissance du secteur agricole (Side, 2013).

Mais la révolution verte a peu touché l'Afrique subsaharienne. L'utilisation de la mécanisation agricole, tout comme les autres innovations (facteurs de production), est faible. L'Afrique détient la plus faible consommation d'énergie et le plus bas niveau d'utilisation des équipements agricole. Selon Side (2013), la principale source d'énergie en agriculture subsaharienne est toujours humaine (65\%), puis animale (25\%) et enfin motorisé (10\%). A titre comparatif, les moteurs réalisent $40 \%$ de la préparation du sol en Asie du Sud et 50\% en Amérique Latine et aux Caraïbes (FAO, 2006, citée par AFD, 2018).

Nous définissons la mécanisation agricole, dans ce papier, comme étant l'utilisation de tout équipement manuel ou tracté par un animal ou motorisé dans les opérations culturales. La mécanisation joue un rôle important dans l'accroissement des superficies cultivées. Par exemple au Sénégal, le passage de la mécanisation manuelle à la traction animale a permis une extension des superficies cultivées. Au Bénin, l'utilisation de la motorisation a provoqué une augmentation des superficies cultivées par exploitation (FARM/FAO, 2015). L'accroissement des rendements induit par la mécanisation est relevé par plusieurs auteurs, Sargent et al. (1981) relève une augmentation de $19 \%$ sur le rendement de l'arachide et de 20 à 30\% sur celui des céréales après un labour profond. Ramond et Tournu (1971) et Pouya et al. (2013) abondent dans le même sens et montrent l'accroissement des rendements avec l'utilisation de la mécanisation. La mécanisation agricole génère aussi un certain niveau de rentabilité des exploitations. Elle se présente comme l'élément le plus efficient par rapport aux travaux avec la force humaine et animale (Soliman, 1992). Campagne (1981) a montré la rentabilité de la mécanisation en insistant sur l'importance de la prestation de service chez les exploitations non équipées. Persoon (1989) a montré un gain de revenu à la suite du passage de la traction animale à la motorisation. Il faut cependant noter que l'utilisation de la mécanisation cause parfois des dommages. Le tassement des sols, provoqué par un émiettement excessif à la suite d'un labour à une vitesse élevée, est relevé par FARM/FAO (2015). Des risques d'érosion (éolienne et hydrique) sont accrus avec l'utilisation de la mécanisation surtout en Afrique de l'Ouest où les sols sont fragiles, peu profonds et soumis à un taux de minéralisation de la matière 
organique élevé (FARM/FAO, ibid.). La faune sauvage est aussi menacée par la mécanisation ; les travaux de récolte coïncident le plus souvent à la période de nidification et d'envol des jeunes oiseaux, ou de mise-bas des mammifères et d'élevage de leurs jeunes (Maillard et al., 2011).

Au Sénégal la demande alimentaire augmente corrélativement à la croissance de la population au taux annuel de 2,5\% (ANSD, 2014). Les faibles rendements agricoles - en moyenne $696 \mathrm{~kg} / \mathrm{ha}$ pour le mil, $806 \mathrm{~kg} / \mathrm{ha}$ pour le sorgho, $1577 \mathrm{~kg} / \mathrm{ha}$ pour le maïs, $818 \mathrm{~kg} / \mathrm{ha}$ pour l'arachide, $540 \mathrm{~kg} / \mathrm{ha}$ pour le niébé (DAPSA, 2017), sont en partie responsables du taux de couverture alimentaire de $38,4 \%$ pour les céréales en 2017. En 2016, ce taux était de l'ordre de 54\%, en se basant sur la norme FAO, stipulant une consommation de $185 \mathrm{~kg}$ de céréales par personne et par an dans les pays du Sahel (DAPSA, 2017 ; USAID, 2017).

Selon l'ISRA (2008), la faiblesse de la productivité agricole de la terre et des rendements est liée à l'irrégularité de la pluviométrie, à la pauvreté des sols, mais aussi à la faible utilisation des technologies de production (semence certifiée, bonnes pratiques culturales ...), à la vétusté du matériel agricole et le faible niveau d'équipement

Dans cet article nous nous intéressons au rôle de la mécanisation dans la production agricole. Comme dans tous les pays d'Afrique Sub-saharienne, au Sénégal, globalement le niveau d'équipements agricoles est insuffisant et la majorité de ces équipements sont anciens et donc peu efficaces (Gouvernement du Sénégal, 2002 ; 2009 ; 2014). Comme ailleurs, les outils manuels et les matériels de traction animale prédominent dans le Bassin arachidier où sont produites plus de $50 \%$ des céréales sèches et des légumineuses du pays (ISRA, 2008).

Nous nous posons la question comment les équipements dans les exploitations impactent les rendements des cultures principales dans le bassin arachidier?

Pour répondre à ces questions, nous utiliserons un modèle économétrique basé sur la fonction de production Cobb-Douglas.

\section{Matériel et méthode}

\section{Cadre théorique de la fonction de Cobb Douglas.}

La fonction de Cobb-Douglas est une fonction largement utilisée en économie comme modèle de fonction de production. Dans ce cadre elle a été proposée par l'économiste américain Paul Douglas et le mathématicien américain Charles Cobb en 1928. Dans leurs travaux, la fonction de production n'est constituée que par deux inputs génériques : le capital et le travail. La fonction caractérise en particulier le lien qui existe entre intrant et extrant.

Fruit (1962) montre que l'intérêt de la fonction est de permettre le calcul des élasticités de la production par rapport aux facteurs de productions. Ainsi, dire que l'élasticité de la production par rapport au travail est $\mathrm{x}$, revient à dire qu'une augmentation de $1 \%$ des quantités de travail déterminera une augmentation de $\mathrm{x} \%$ des quantités produites.

D'abord utilisée pour la production manufacturière américaine, la fonction de Cobb Douglas a été adaptée au secteur agricole. Les fonctions de production sont utilisées par plusieurs auteurs pour déterminer l'impact d'une technologie. Soliman (1992), étudiant le contexte de l'agriculture égyptienne, considère la fonction de production Cobb-Douglas comme la plus pertinente pour 
Effet de la mécanisation sur la productivité des exploitations agricoles dans...

déterminer l'impact de la mécanisation. Soliman a utilisé les fonctions de réponse aux cultures estimées pour dériver la fonction de courbes Isoquant et a appliqué les fonctions Isoclines aux coûts de fonctionnement des machines et au taux de salaire du marché non subventionnés. Son modèle traite des paramètres économétriques, notamment, la spécification du modèle, la multi colinéarité et la qualité de l'ajustement. La fonction de production Cobb-Douglas permet une utilisation simple des facteurs de production (Chisango et Obi, 2012).

\section{Zone d'étude}

Les communes, objet d'étude, sont situées dans le Bassin arachidier, dans les régions de Kaolack et Kaffrine (figure 1).

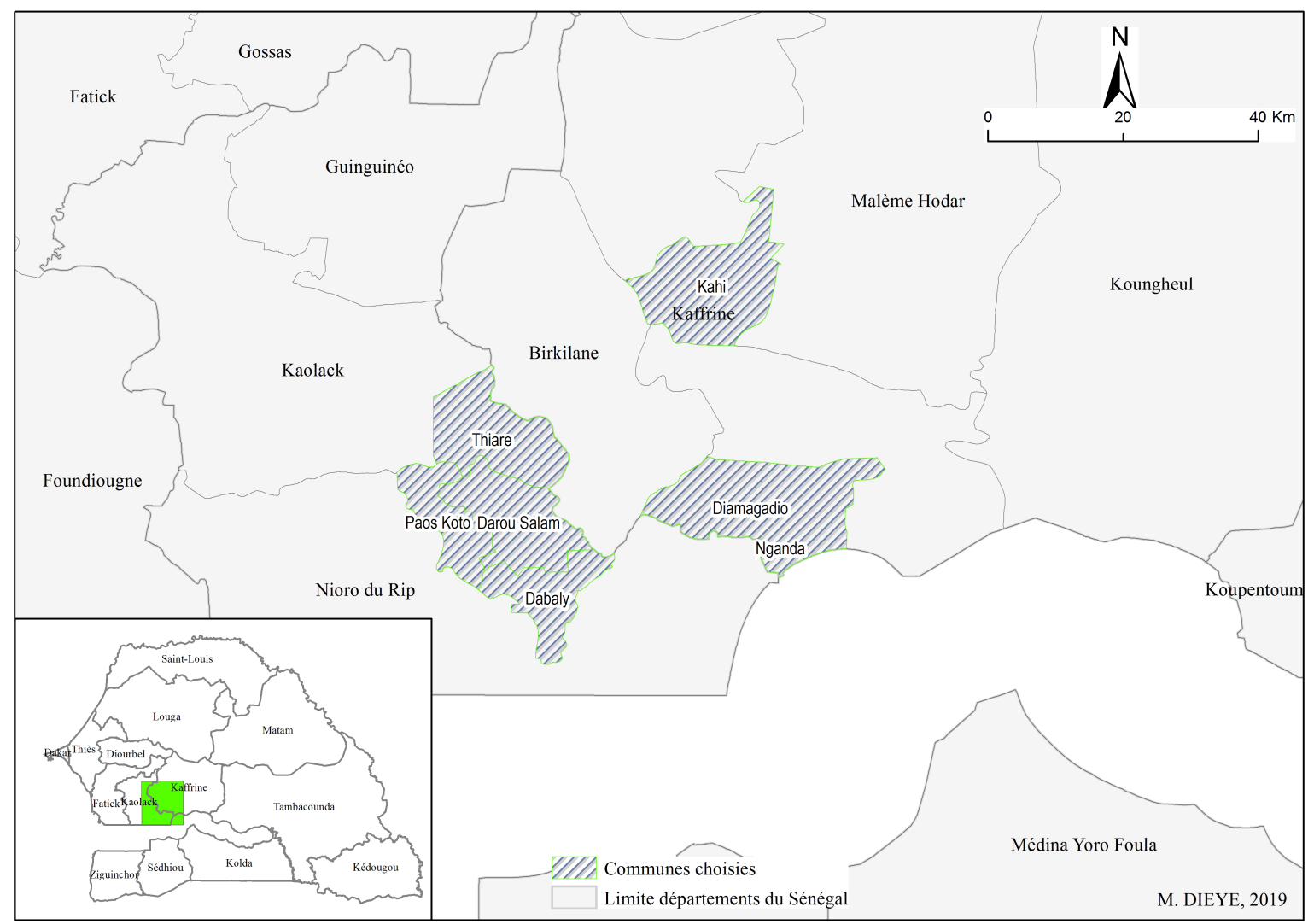

Figure 1 : Cartographie de la zone d'étude

Cette étude s'inscrit dans le cadre d'un projet coordonné par l’Association Sénégalaise pour la Promotion du Développement à la Base (ASPRODEB) à l'attention des membres de cette dernière dans le Bassin Arachidier. Le projet est intitulé "Diffusion à grande échelle de kits de matériels agricole et de transformation des produits agricoles". ASPRODEB est une organisation paysanne et son Réseau National des Coopératives de Producteurs de Semences (RNCPS), dans le Bassin arachidier est l'organisation paysanne (OP) prise en compte par l'étude.

Le Bassin arachidier a été au cœur des interventions de l’État en termes de mécanisation (Dieng, 2006). Il est caractérisé par un système de production mil/arachide, dans lequel la traction animale est très utilisée pour les semis à sec (mil, parfois sorgho) et en humide (arachide, maïs, niébé, etc.), 
les sarclages, les buttages et le soulevage de l'arachide, mais aussi le transport (Havard, 1986 ; $1987 ; 1988$ et ISRA, 2008).

Les spéculations les plus cultivées sont l'arachide, le mil, le maïs, le sorgho, le niébé, la pastèque, l'oseille de Guinée et les cultures horticoles comme la tomate, le piment, l'aubergine, les laitues, le gombo. L'étude ne s'intéresse qu'aux spéculations majeures à savoir l'arachide, le mil et le maïs. Selon les données de la Direction de l'Analyse de la Prévision et des Statistiques Agricoles (DAPSA) de 2013, l'arachide occupe 46\% des superficies emblavées dans le Bassin arachidier, le mil 38\%, le maïs $5 \%$ et le sorgho $3 \%$.

Les opérations culturales (préparation du sol, semis, désherbage, récolte ...) sont généralement effectuées par des outils de traction animale tirés par les chevaux et les ânes, et beaucoup plus rarement par une paire de bœufs. Le travail du sol est superficiel et généralement réalisé avec une houe sine ou une houe occidentale équipée de trois dents avec des socs patte d'oie ou de scarifiage. Les semis de mil, sorgho, maïs, arachide, niébé sont effectués avec le semoir Super-Eco. Les houes, occidentale ou sine, sont utilisées pour le désherbage entre les lignes, d'autres outils manuels sont aussi utilisés (hiler, daba, ngonsi, ...) pour la même opération sur la ligne de semis. Les céréales sont récoltées manuellement avec un couteau ou d'autres petits outils à lame. Le soulevage de l'arachide est effectuée avec une souleveuse à lame tirée par des animaux de trait; le ramassage de l'arachide et la mise en moyettes ou en meules est manuelle. Le transport des récoltes s'effectue avec les charrettes à traction animale.

\section{Échantillonnage}

Les producteurs ciblés sont membres de l'ASPRODEB. Celle-ci fournit aux coopératives les services de renforcement de capacités techniques et organisationnelles, de gestion financière et d'appuiconseil. Elle constitue une courroie de transmission pour les coopératives. Elle est bien structurée et bien représentée dans toutes les régions du pays avec plus 26 fédérations de coopératives et groupements. Le Réseau National des Coopératives de Producteurs de Semences (RNCPS), dans le Bassin arachidier est l'organisation de producteurs membres de l'ASPRODEB prise en compte par l'étude. Ce réseau regroupe plus de 1900 membres : communes de Paoskoto et de Thiaré (Kaolack), de Nganda et Kahi (Kaffrine).

Les exploitations familiales, porte d'entrée pour l'étude, sont choisies suivant leur appartenance aux OP retenues. Un échantillonnage probabiliste aléatoire simple a été appliqué sur la base des membres des groupements qui constituent notre base de sondage. La méthode s'annonce comme suit : 


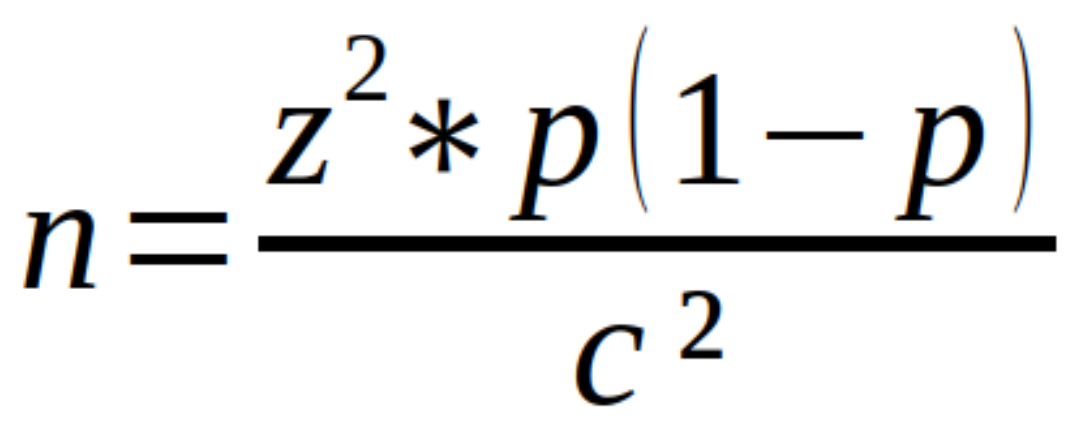

Avec : n : la taille de l'échantillon; $\mathrm{z}$ : niveau de confiance ; p : la probabilité de réalisation de l'événement ; $\mathrm{c}$ : la marge d'erreur ;

Cet échantillon est corrigé avec la formule suivante :

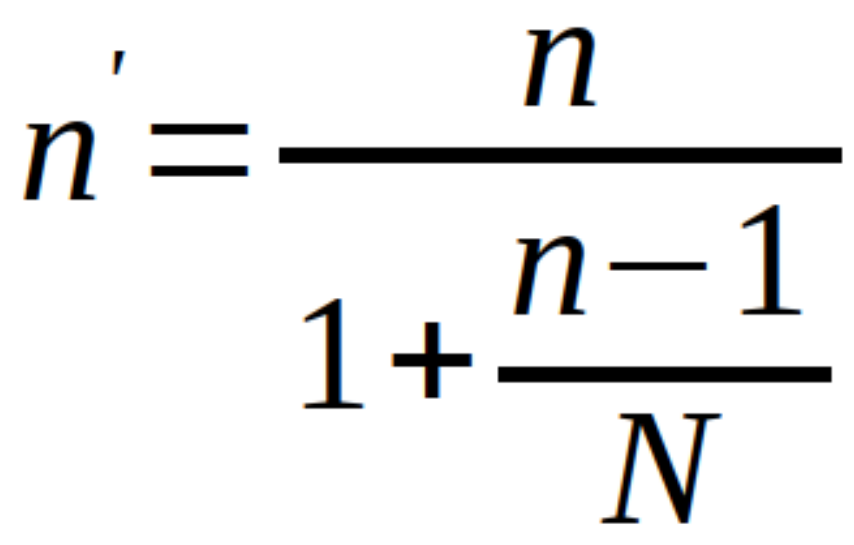

Avec : n' : Taille de l'échantillon corrigé ou final ; $\mathrm{N}$ : Taille de la population

Ainsi, un échantillon de 321 ménages a été retenu dans quatre départements et regroupant quatre coopératives (tableau 1). 
Tropicultura Tropicultura 2295-8010 Volume 39 (2021) Numéro 1, 1680

Tableau 1 : Caractéristiques de l'échantillon

\begin{tabular}{|c|c|c|c|c|}
\hline Région & Département & Coopératives & Nombre de membres & Echantillon \\
\hline \multirow[t]{2}{*}{ Kaolack } & Nioro & Coopérative de Paoskoto & 463 & 76 \\
\hline & Kaolack & Coopérative Thiaré & 374 & 62 \\
\hline \multirow[t]{2}{*}{ Kaffrine } & Malem Hoddar & Coopérative de Nganda & 312 & 51 \\
\hline & Kaffrine & Coopérative de Kahi & 800 & 132 \\
\hline Total & 4 & 4 & 1949 & 321 \\
\hline
\end{tabular}

\section{Collecte des données}

Un guide et un questionnaire ont été élaborés pour collecter des données aussi bien quantitatives (superficie emblavée, nombre d'équipements agricoles, la main d'œuvre, les intrants, ...) que qualitatives auprès des producteurs (l'organisation de la production, la gestion des équipements...). Les données sur les facteurs de production (intrants et équipement agricole), la main d'œuvre, les superficies emblavées et la production alimentent notre modèle. Les données collectées concernent le parc de matériel et son utilisation par les producteurs. Tous les aspects liés aux facteurs de production et aux revenus (production, vente, prix, etc.), sont aussi renseignés.

\section{Analyse des données}

Pour mesurer l'impact de la mécanisation plusieurs approches sont utilisées par les auteurs. La perception des poducteurs est utilisée par Pouya et al. (2013), pour évaluer l'impact d'innovations agricoles introduites. Des méthodes expérimentales comme la randomisation peuvent aussi être utilisées. En effet, lorsqu'elles sont appliquées correctement, elles produisent la meilleure estimation de l'impact d'un traitement (Duflo et al, 2007 ; Diaz et Handa, 2004.). Les méthodes non expérimentales sont une alternative aux méthodes expérimentales. D'après Diaz et Handa (2004), leurs résultats dépendent d'hypothèses et les principales méthodes non expérimentales sont : la méthode d'appariement, la double différence et les variables instrumentales. Des méthodes paramétriques sont aussi utilisées dans l'évaluation d'impact. Les principales approches utilisées sont l'approche économétrique à travers les simulations économétriques et la programmation mathématique (Van Imhoff Evert, 1997).

Dans cette étude l'approche paramétrique est utilisée à travers la fonction de production. La productivité de la terre, mesurée par le rendement est l'indicateur utilisé dans cette étude pour mesurer l'impact de la mécanisation. La variation des rendements dans une localité est considérée comme indicateur d'une amélioration de l'efficience économique. Dans leur fonction de production, la valeur monétaire de tous les équipements, machines et bâtiments a été intégré au capital.

Leur fonction de production de type Cobb-Douglas est énoncée comme suit : 


\section{$Y=A K^{\alpha} L^{\beta}$}

Avec : $\mathrm{Y}=$ niveau de production $; \mathrm{K}=$ capital $; \mathrm{L}=$ travail $; \mathrm{A}$, $\alpha$ et $\beta$ sont des constantes.

Cette fonction intégre les variables capital et travail (main d’œuvre). Le capital peut être décomposé en capital foncier, humain, équipement... Ainsi, le rendement est régressé sur les facteurs de production (régression multiple) afin de déterminer ceux qui influencent le rendement. Cette régression a été faite avec la fonction de production Cobb Douglas linéarisée. Ce qui donne :

\section{$\log (Y)=\beta_{0}+\beta_{1} \log (X \| 1)+\beta_{2} \log (X \| 2)+\ldots+\beta_{n} \log (X \| n)+\varepsilon$}

Avec Y : rendement ; X : les facteurs de production et l'erreur

Dans leur étude sur l'efficacité technique dans certains États de l'Inde, Ghoshal and Goswami (2017) ont utilisé la technique de la fonction de production stochastique avec Cobb Douglas sur les variables suivantes : crédit institutionnel, superficie nette irriguée, consommation d'engrais et de pesticides, taux d'alphabétisation en milieu rural, niveau de l'enseignement technique dans les zones rurales etc. La superficie cultivée, la superficie irriguée effective, l'utilisation d'engrais chimiques, la puissance des machines agricoles, la consommation d'électricité rurale et la maind'œuvre sont considérées comme des variables indépendantes de la fonction de production de Cobb-Douglas, par Yuan (2011) dans son étude sur l'analyse de la relation entre la production agricole et les facteurs d'intrants dans la province de Hebei. Afin d'analyser les processus de production agricole au Sri Lanka, la fonction de production de Cobb-Douglas a été utilisée par Dhannasiri et Datye (2011) pour mesurer la contribution marginale des intrants à la production agricole. Les variables utilisées sont les coûts des intrants tels que les engrais, semences, produits chimiques, main-d'œuvre, transport, taxes, etc. 
Tropicultura Tropicultura 2295-8010 Volume 39 (2021) Numéro 1, 1680

Tableau 2 : Récapitulatif des indicateurs utilisés par certains auteurs

\begin{tabular}{|c|c|c|}
\hline Auteur & Année & Indicateurs \\
\hline Ghoshal ey Goswami & 2017 & $\begin{array}{l}\text { - le crédit institutionnel } \\
\text { - la superficie nette irriguée } \\
\text { - la consommation d'engrais et de pesticides } \\
\text { - le taux d'alphabétisation } \\
\text { - le niveau de l'enseignement technique }\end{array}$ \\
\hline Yuan & 2011 & $\begin{array}{l}\text { - La superficie cultivée } \\
\text { - la superficie irriguée effective } \\
\text { - l'utilisation d'engrais chimiques } \\
\text { - la puissance des machines agricoles } \\
\text { - la consommation d'électricité rurale } \\
\text { - la main-d'œuvre }\end{array}$ \\
\hline Dhannasiri et Datye & 2011 & $\begin{array}{l}\text { - les coûts des engrais } \\
\text { - les coûts des semences } \\
\text { - les coûts produits pesticides } \\
\text { - les coûts de la main-d'œuvre } \\
\text { - les coûts du transport } \\
\text { - les coûts des taxes }\end{array}$ \\
\hline
\end{tabular}

De ces exemples, nous proposons que compte tenu des spécificités des systèmes de production et des intrants utilisés par les exploitations agricoles de notre zone d'étude, notre fonction de production se présente comme suit :

$\log ($ rendement $)=\beta_{0}+\beta_{1} \log ($ semence $)+\beta_{2} \log ($ fertilisation $)+\beta_{3} \log ($ main-œuvre $)+\beta_{4} \log$ $($ mecanisation $)+\beta_{5} \log ($ location terre $)+\beta_{6} \log ($ animaux-trait $)+\beta_{7}$ genre $+\beta_{8} \log (\hat{a g e})+\beta 9$ niveau instruction $+\beta_{10}$ formation professionnelle $+\varepsilon$

Pour la mécanisation, les charges d'amortissement, de location et d'achats de petits matériels ont été prises en compte dans l'équation de régression. Concernant les animaux de trait, les charges d'amortissement et d'entretien (alimentation et soins sanitaires) ont été intégrées dans les calculs. Pour éviter aussi les biais liés à l'effet de taille, c'est-à-dire des charges corrélées à la taille de 
Effet de la mécanisation sur la productivité des exploitations agricoles dans...

l'exploitation, tous les facteurs sont ramenés à l'hectare.

\section{Résultats}

Le coefficient d'un facteur de production (semences, engrais, etc.) est significatif lorsque la variable explicative correspondante contribue de façon significative à l'explication de la variable endogène. La significativité d'un coefficient est testée à partir du t de Student. On teste l'hypothèse d'un coefficient nul contre l'hypothèse alternative d'un coefficient différent de zéro. Selon le test de Student le coefficient sera significatif si la probabilité est inférieure au seuil de $5 \%$. Cette probabilité est calculée sur la base de l'hypothèse de normalité des termes d'erreur.

\section{Effet sur le rendement en arachide}

Globalement, les résultats de la régression montrent que sur l'arachide, les facteurs de production - semence, engrais, équipement agricole 1 , et nombre d'actifs impactent significativement le rendement avec des coefficients respectifs de 0,$1549519 ; 0,3420537 ; 0,0489993$ et 0,3627825 (Tableau 2). Ces facteurs sont significatifs au seuil de $1 \%$ excepté les semences significatives au seuil de $5 \%$.

Les équipements agricoles mobilisés actuellement par les exploitations agricoles sur la culture d'arachide impactent faiblement le rendement. Le modèle montre que l'augmentation du rendement ne suit pas celle sur l'investissement sur les équipements. Mais on peut se poser la question : avec un meilleur équipement des exploitations l'effet sur le rendement ne serait-il pas plus conséquent?

\section{Effet sur le rendement en mil}

Le rendement en mil est fonction de l'engrais, de l'équipement agricole et de l'âge du producteur (Tableau 2). L'engrais et l'âge du producteur sont significatifs au seuil de 1\%. En revanche, l'équipement agricole est significatif au seuil de $5 \%$. Les simulations ont montré que la mécanisation mobilisée sur la culture du mil, même doublée entraînerait un surplus de rendement de mil de moins de $11 \%$. Ce qui montre que l'acquisition de rendements substantiels par l'accroissement de la mécanisation n'est pas automatique et certaines opérations impactant plus le rendement sont à prioriser dans la stratégie d'équipement des exploitations agricoles (mettre en avant les matériels de préparation du sol, semis et du désherbage).

Effet sur le rendement en maïs

Le modèle montre que sur la culture du maïs, la quantité de semences, l'âge des producteurs, le niveau universitaire plutôt que le niveau primaire, et le nombre d'actifs impactent le rendement de maïs au seuil de $5 \%$ avec des coefficients respectifs de 0,$5898171 ; 1,867215 ;-1,5686$ et 0,9172302 (Tableau 2).

Les équipements actuels, mobilisés sur la culture de maïs par les exploitations agricoles, a un impact peu significatif sur le rendement. Ceci peut s'expliquer par la faiblesse des équipements mobilisés par les exploitations agricoles sur cette culture. D'où la nécessité de renforcer l'utilisation des équipements agricoles dans la culture de maïs dans les opérations comme le labour le semis et le sarclage. 


\section{Discussion}

Les équipements utilisés actuellement par les exploitations ont un impact significatif au seuil de $1 \%$ pour le rendement d'arachide et $5 \%$ pour celui du mil. Si l'investissement sur les équipements agricoles est amélioré, les rendements seront augmentés de 5 et $11 \%$ respectivement sur l'arachide et le mil. Toutefois, ces équipements sont peu significatifs sur la culture du maïs.

L'impact des équipements utilisés sur certaines cultures est corroboré par les résultats de Havard (1997) après exploitation des données de Nicou et Le Moigne (1990) en station. Il montre que certaines opérations comme le labour procure des gains de rendement qui peuvent aller jusqu'à $28 \%$ sur les céréales et $20 \%$ sur les légumineuses. Sargent et al. (1981) abondent dans le même sens et relèvent une augmentation de 19\% du rendement d'arachide après un labour profond.

Les effets de la mécanisation agricole sont plus visibles sur l'extension des superficies, l'allègement des charges et l'accroissement de la productivité du travail (AFD, 2018 ; Side, 2013). Cependant, les effets sur les rendements sont non négligeables. Le retard sur les opérations culturales occasionne des pertes de rendement que peut éviter la mécanisation. Ces résultats montrent tous qu'il y a un gain de rendement aussi minime soit-il avec l'utilisation de la mécanisation sur la production d'arachide. Cependant, un écart existe entre nos résultats (données de terrains) et ceux des auteurs précédents. Les écarts observés entre les résultats peuvent s'expliquer en partie par le fort taux de matériels à usage manuels, la vétusté et la faiblesse de la traction animale dans les exploitations agricoles familiales.

Sur les cultures céréalières, Ramond et Tournu (1974) ont montré un accroissement des rendements pouvant atteindre $50 \%$ avec l'effet de la mécanisation (labour profond) dans le Sine Saloum alors que nos résultats ne dépassent pas $11 \%$. Ceci peut s'expliquer par le fait qu'en milieu paysan les opérations peuvent ne pas être effectuées avec la même efficacité qu'en station. 
Effet de la mécanisation sur la productivité des exploitations agricoles dans...

Tableau 3 : Déterminants des rendements en arachide, mil, maïs et riz

\begin{tabular}{|c|c|c|c|}
\hline VARIABLE & $\begin{array}{l}\text { LOG (REND } \\
\text { ARACHIDE) }\end{array}$ & $\begin{array}{c}\text { LOG (REND } \\
\text { MAIS) }\end{array}$ & $\begin{array}{c}\text { LOG (REND } \\
\text { MIL) }\end{array}$ \\
\hline Stat. Fisher & 4.95 & 4.63 & 181.21 \\
\hline$P$-value & 0.0000 & 0.0002 & 0.0000 \\
\hline \multirow[t]{2}{*}{ Main d'œuvre } & $-0,0291576$ & $-0,7412611$ & 0,4942086 \\
\hline & $(0,1862466)$ & $(0,6423435)$ & $(0,4103276)$ \\
\hline \multirow[t]{2}{*}{ Quantité d'engrais } & $0,3420537 * * *$ & 0,1939898 & $0,4575098 * * *$ \\
\hline & $(0,111361)$ & $(0,1800425)$ & $(0,1659176)$ \\
\hline \multirow[t]{2}{*}{ Quantité de semence } & $0,1549519 * *$ & $0,5898171^{* *}$ & 0,4101722 \\
\hline & $(0,0730718)$ & $(0,2722254)$ & $(0,3976559)$ \\
\hline \multirow[t]{2}{*}{ Coût location terre } & $-0,0232249$ & $-0,0145045$ & $-0,0168287$ \\
\hline & $(0,0180175)$ & $(0,0494699)$ & $(0,0412964)$ \\
\hline \multirow[t]{2}{*}{ Coût équipement } & $0,0489993 * * *$ & 0,037402 & $0,1057298 * *$ \\
\hline & $(0,0178638)$ & $(0,0669469)$ & $(0,0512259)$ \\
\hline \multirow[t]{2}{*}{ Charges, animaux de trait } & 0,0316541 & 0,1324792 & 0,0500773 \\
\hline & $(0,0283354)$ & $(0,0879449)$ & $(0,0531369)$ \\
\hline \multirow[t]{2}{*}{ Genre (femme) } & 0,0823319 & $-0,2293492$ & 0,1669226 \\
\hline & $(0,2002417)$ & $(0,5582469)$ & $(0,5147934)$ \\
\hline \multirow[t]{2}{*}{ Age } & $-0,1923341$ & $1,867215^{* *}$ & $0,94669 * * *$ \\
\hline & $(0,3160947)$ & $(0,7775181)$ & $(0,2436354)$ \\
\hline \multirow[t]{2}{*}{ Nombre d'actif } & $0,3627825 * * *$ & $0,9172302 *$ & 0,0779728 \\
\hline & $(0,1260807)$ & $(0,4689543)$ & $(0,2839045)$ \\
\hline \multirow[t]{2}{*}{ Niveau d'instruction secondaire } & & 0,0615319 & $-0,2447945$ \\
\hline & & $(0,3859767)$ & $(0,3317345)$ \\
\hline \multirow[t]{2}{*}{ Niveau d'instruction universitaire } & & $-1,5686 * *$ & $-0,3985636$ \\
\hline & & $(0,6272201)$ & $(0,5179797)$ \\
\hline \multirow[t]{2}{*}{ Formation professionnelle } & & $-1,372948$ & 0,0367908 \\
\hline & & $(0,8758593)$ & $(0,6475286)$ \\
\hline \multirow[t]{2}{*}{ Constante } & $4,464429 * * *$ & $-5,948287^{*}$ & \\
\hline & $(1,229653)$ & $(3,391707)$ & \\
\hline Observations & 296 & 47 & 66 \\
\hline$R$-squared & 0.1347 & 0.6203 & 0.9758 \\
\hline Standard errors in parentheses & \multicolumn{3}{|c|}{$* * * \mathrm{p}<0.01, * * \mathrm{p}<0.05, * \mathrm{p}<0.1$} \\
\hline
\end{tabular}

\section{Conclusion}

L'étude a mis en évidence que les équipements utilisés actuellement dans les exploitations agricoles du bassin arachidier n'améliorent pas les rendements des cultures. Quelles que soient la culture dans le Bassin arachidier, les équipements actuels, même doublés, ne favorisent pas un accroissement significatif du rendement des cultures. Ainsi, la stratégie de mécanisation doit être orientée objectif de productivité et non sur une accumulation d'équipement.

Il découle principalement de l'étude que des efforts considérables doivent être entrepris par l'Etat et les autres acteurs au profit des producteurs non seulement sur les équipements mais aussi 
sur les autres facteurs de production qui impactent le rendement tels que les intrants, la main d'œuvre et le niveau d'instruction. Le développement de l'agriculture à travers l'accroissement de la productivité agricole de la terre passe nécessairement par une maitrise de ces facteurs.

Le facteur mécanisation impacte significativement le mil et l'arachide. Cependant, d'autres facteurs de productions (l'engrais, les semences, la main d'œuvre) montrent aussi des significativités différentes sur le rendement selon les cultures.

Les cultures étudiées dépendent aussi de la pluviométrie, de la technicité des producteurs, de la composition physico-chimique des sols entre autres facteurs. Ces paramètres ne sont pas pris en compte dans cette étude.

\section{Remerciement}

Sincères remerciements au regretté Michel Havard (chercheur au CIRAD) qui nous a quitté sur la pointe des pieds. Nous aurions souhaité que l'aboutissement de ce travail, que tu as accompagné depuis le début, te trouve parmi nous. Reçois de là où tu es notre grande reconnaissance.

Tu resteras à jamais dans nos cœurs.

\section{Bibliographie}

1. AFD, 2018, Mécanisation agricole et politiques publiques dans les pays en développement. AFD, Paris, France, 25p.

2. ANSD, 2014, Recensement Général de la Population et de l'Habitat, de l'Agriculture et de l’Elevage. Agence Nationale de la Statistique et de la Démographie, Dakar Sénégal, 417p.

3. Berthelier P. Lipchitz A., 2005, Quel rôle joue l'agriculture dans la croissance et le développement? Revue Tiers Monde, $\mathrm{n}^{\circ}$ 183, 603-624pp. DOI 10.3917/rtm.183.0603

4. Blancard S. Boussemart J. P. Flahaut J. et Lefer H. B., 2013, Les fonctions distances pour évaluer la performance productive d'exploitations agricoles, Économie rurale [En ligne], $\mathrm{N}^{\circ}$ :334, mis en ligne le 15 mars 2015, consulté le 19 avril 2019. URL : http://journals.openedition.org/economierurale/3887 ; DOI : 10.4000/ economierurale.3887

5. Chisango et Obi, 2012, Efficiency Effects Zimbabwe's Agricultural Mechanization and Fast Track Land Reform Programme: A Stochastic Frontier Approach. Department of Agricultural Economics and Extention University of Fort Hare, Alice, 23p.

6. Colin J.P., sd, Eléments d'analyse micro-économique de la production agricole. In : Robineau Claude (ed.), Penouil M. (pref.) Les terrains du développement : approche pluridisciplinaire des économies du Sud. Paris : ORSTOM, pp. 129-138.

7. DAPSA, 2017, Revue 2017 du sous-secteur agricole, Ministère de l'Agriculture et de l’Equipement Rural. Dakar, Sénégal, 40p.

8. Dhannasiri L. M. \& Datye V. S., 2011, Application of Cobb-Douglas Function for Analyzing the Process of Agricultural Production: A Case Study from Sri Lanka. Transactions of the 
Effet de la mécanisation sur la productivité des exploitations agricoles dans...

Institute of Indian Geographers, Vol. 33. No. 2; pp. 251-263.

9. Dieng A., 2006, Impacts des politiques agricoles sur l'offre céréalière au Sénégal, de 1960 à 2003 : Évaluation à partir d'un modèle d'analyse statistique par zones agro-écologiques. Thèse Pour obtenir le grade de Docteur en sciences économiques : UFR des Sciences Economiques. Bourgogne : Université de Bourgogne, France, 313p.

10. Fruit R., 1962, La fonction de production de Cobb-Douglas. In: Revue économique, volume $13, \mathrm{n}^{\circ} 2$, 1962. pp. 186-236; doi : 10.2307/3498284

11. FAO, 2000, Production et productivité agricoles dans les pays en développement, In La situation mondiale de l'alimentation et de l'agriculture 2000, Fao, Rome, Italie. http://www.fao.org/3/x4400f/x4400f12.htm

12. Ghosal P. \& Goswani B., 2017, Cobb-Douglas Production Function for Measuring Efficiency in Indian Agriculture: A Region-wise Analysis. Economic Affairs, Vol. 62, No. 4, pp. 573-579, DOI: 10.5958/0976-4666.2017.00069.9

13. Gouvernement du Sénégal, 2002, Document de Stratégie de Réduction de la Pauvreté (DSRP). Dakar, Sénégal, 77p.

14. Gouvernement du Sénégal, 2009, Programme National d'Autosuffisance en Riz (PNAR). Stratégie nationale de développement de la riziculture, Dakar, Sénégal. 26p.

15. Gouvernement du Sénégal, 2014, Plan Sénégal Emergent (PSE), Dakar, Sénégal. 167p.

16. Havard M., 1997, Impact de l'utilisation de la traction animale sur les productions agricoles. Exemples en Afrique francophone subsaharienne. Cours, programme D.E.A, Amélioration des systèmes de productions agricoles, Faculté Universitaire des Sciences Agronomiques de Gembloux, Belgique. 21p.

17. Havard M., 1986, Les Caractéristiques, la gestion et la maintenance des outils manuels et de culture attelée sur l'unité expérimentale de Thyssé-Kayemor, Institut Sénégalais de Recherches Agricoles ISRA, Département Système et Transfert, Dakar, Sénégal. 74p.

18. Havard M., 1987, La mécanisation agricole face à un changement de politique d'équipement, Institut Sénégalais de Recherches Agricoles, Direction de Recherches sur les Systèmes Agraires, Dakar, Sénégal. 15 p.

19. Maillard J.F. Suffran Y. et Omnes F., 2011, Machinisme agricole et faune sauvage, Faune Sauvage, $\mathrm{N}^{\circ} 291$, 2e trimestre 2011, pp. 49-54

20. Mbengue H.M. \& Havard M., 1989, Une réflexion insuffisante sur les orientations de la mécanisation au Sénégal. Quelques exemples concrets. Economie de la mécanisation en régions chaudes. Actes du IXème séminaire d'économie rurale, 12 au 16 septembre 1988, CIRAD, Montpellier, France.

21. ISRA, 2008, Caractérisation et typologie des exploitations agricoles familiales du Sénégal, Tome 3 : Bassin arachidier, vol 8 N³, ISRA, Dakar, Sénégal. 30p.

22. Mazoyer M. \& L. Roudart L., 2008, "Histoire des agricultures", Encyclopaedia universalis. 
23. Ramond C.et Tournu G., 1974, Unités expérimentales du Sine Saloum. Analyse des rendements parcellaires en milieu paysan. Résultats obtenus pendant la campagne 1972. Document technique et de recherche. Bambey : CNRA, Sénégal. 76 p.

24. Sargent M. Litche J. Matlon P. \& Bloom R., 1981, An Assessment of Animal Traction in Francophone West Africa. Dep. Agr. Econ. African Rural Economy Work Pap. No. 34., Michigan State University.

25. Side C.S., 2013, Stratégie de mécanisation de l'agriculture familiale en Afrique SubSaharienne. Inclus Étude de cas du Burkina-Faso. Mastère Spécialisé en Innovations et Politiques pour une alimentation durable (IPAD). SupAgro, AFD, Montpellier, France, $106 \mathrm{p}$.

26. Soliman I., 1992, "Agricultural mechanization and economic efficiency of agricultural production in Egypt", international conference on agricultural engineering \& rural development, volume 1, Beijing, Chine. pp. 54-59

27. USAID, 2017, Etude de la consommation des céréales de Base au Sénégal, Feed the Future Sénégal Naatal Mbay, 126 p.

28. Yuan Z., 2011, Analysis of agricultural input-output based on Cobb-Douglas production function in Hebei Province, North China. African Journal of Microbiology Research, Vol. 5(32), pp. 5916-5922, DOI: 10.5897/AJMR11.961

\section{Notes}

1 Par équipement agricole nous entendons les charges d'amortissement, de location et d'achats de petits matériels

PDF généré automatiquement le 2023-04-26 14:34:10

Url de l'article : https://popups.uliege.be/2295-8010/index.php?id=1680 